\title{
Trip Around the HMPerceptron Algorithm: Empirical Findings and Theoretical Tenets
}

\author{
Roberto Esposito and Daniele P. Radicioni \\ Dipartimento di Informatica, Università di Torino \\ \{esposito,radicion\}@di.unito.it \\ Corso Svizzera 185, 10149 - Torino
}

\begin{abstract}
In a recent work we have carried out CarpeDiem, a novel algorithm for the fast evaluation of Supervised Sequential Learning (SSL) classifiers. In this paper we point out some interesting unexpected aspects of the learning behavior of the HMPerceptron algorithm that affect CarpeDiem performances. This observation is the starting point of an investigation about the internal working of the HMPerceptron, which unveils crucial details of the internal working of the HMPerceptron learning strategy. The understanding of these details, augment the comprehension of the algorithm meanwhile suggesting further enhancements.
\end{abstract}

\section{Introduction: the SSL task}

In the supervised learning framework examples are assumed to be drawn independently and identically from some joint distribution $P(x, y)$. While this assumption is fulfilled in many application fields, there are many others where the classified data has a sequential structure (i.e., nearby $x$ elements are likely to have correlated classifications) that must be exploited in order to obtain accurate classifiers. To analyse web logs, to recognize speech or handwriting, to understand music are all tasks where this correlation exists and is essential to the classification purposes.

Within the broader field of learning systems for sequential data, let us consider the Supervised Sequential Learning (SSL) task: in this particular problem, each observation in the sequence is associated with an individual label. The goal of SSL systems is to learn how to best predict the sequence of labels, given the sequence of observations. More formally, the SSL task can be specified as follows [1:

Given: A set $L$ of training examples of the form $\left(X_{m}, Y_{m}\right)$, where each $X_{m}=$ $\left(x_{m, 1}, \ldots, x_{m, T_{m}}\right)$ is a sequence of $T_{m}$ feature vectors and each $Y_{m}=\left(y_{m, 1}\right.$, $\left.\ldots, y_{m, T_{m}}\right)$ is a corresponding sequence of class labels, $y \in\{1, \ldots, K\}$.

Find: A classifier $H$ that, given a new sequence $X$ of feature vectors, predicts the corresponding sequence of class labels $Y=H(X)$ accurately.

The SSL problem has been approached with many different techniques. Among others, we recall Sliding Windows [1], Hidden Markov Models 2], Maximum Entropy Markov Models [3, Conditional Random Fields [4], and the HMPerceptron algorithm [5]. 
The HMPerceptron has been defined within the boolean features framework $[3$. In this setting, the learnt classifier is built in terms of a linear combination of boolean features. Each feature reports about a salient aspect of the sequence to be labelled in a given time instant. More formally, given a time point $t$, a boolean feature is a $1 / 0$-valued function of the whole sequence of feature vectors $X$, and of a restricted neighborhood of $y_{t}$. The function is meant to return 1 if the characteristics of the sequence $X$ around time step $t$ support the classifications given at and around $y_{t}$.

In general, the labeling of an entire sequence $X$ may show dependences among labels which extend on the whole labeling. In this case, the labeling task must take into account every possible sequence of labels, thus resulting in a $\Theta\left(K^{T}\right)$ complexity. However in practice it is possible to evaluate $H$ in polinomial time by making some simplifying assumption. In particular, assuming a first order Markov property allows evaluating $H$ in quadratic time by means of the Viterbi algorithm [6]. The Viterbi algorithm is thereby crucial to many SSL systems, in that most state-of-the-art methods for dealing with the SSL task rely on the Viterbi algorithm for classifying and/or learning purposes.

Unfortunately even the drastic reduction in complexity achieved by Viterbi's algorithm is not always sufficient in many important application domains. In particular, when the number of possible labels is large (more than few tens) as in some web-logs related tasks [7] or in analyzing music [8], the classification times can grow prohibitively high. In order to allow sub-quadratic evaluation of sequential classifiers, we have recently proposed the CarpeDiem 9] algorithm, which returns the same results as Viterbi, meanwhile allowing for significant time savings.

In the present paper we provide an experimental assessment of its functioning and of its peculiarities. We start by expanding on the time saving that can be obtained when CarpeDiem is used instead of Viterbi in the HMPerceptron learning algorithm. We then recall the functioning of CarpeDiem, and introduce further experiments. The new experimentation shows that - to a good extent, unexpectedly - by increasing the size of the training set, and/or the number of learning iterations, we badly affect the classification time. The rest of the paper is devoted to investigate this phenomenon and to draw consequences about the way learning works.

\section{Review of Past Results}

The main idea underlying CarpeDiem stems from noting that in many application domains classifying an item at time $t$ not always requires knowledge about both previous and current labels, $y_{t-1}, y_{t}$. On the contrary, very often the characteristics of the item are, by themselves, very relevant for predicting $y_{t}$. Then, we identify two kind of features: the vertical features that do not require to know the previously predicted label -and work, thus, under a zero-order Markov assumption-, and the horizontal features that do need it -thereby working under a first-order Markov assumption. For instance, in speech recognition the features 
that report about the current phoneme are what we refer to as vertical features. By contrast, we call horizontal features those ones taking into consideration the previously predicted phoneme. For instance, one may want to discourage the prediction of phoneme $/ \mathrm{r} /$ after phoneme /s/. In such application vertical features provide much information about the classification: based on vertical features inspection, it may happen that phoneme $/ \mathrm{d} /$ is confused with $/ \mathrm{t} /$, but rarely this happens with $/ \mathrm{t} \int /$. In other words, one would expect that after inspecting vertical information, the uncertainty about the correct label sensibly decreases until only few of the initial guesses are left as probable.

CarpeDiem works by exploiting vertical information to avoid evaluating the more costly horizontal features. In the best case, it scans only the most promising node for each layer (instead of all the nodes, like Viterbi). In such a case, the cost of the algorithm would be $\Theta(K \log (K) T)$, where the factor $K \log (K)$ is due to the time spent for sorting the nodes in each layer ${ }^{1}$ In the worst case (no vertical features), the algorithm has a complexity of $K^{2} T$, that is, the algorithm is never asymptotically worse than Viterbi algorithm [9].

In the following and throughout the paper, we will report and elaborate about experimental performances obtained on tonal harmony analysis. The task is briefly introduced in the following.

\subsection{Test domain: Harmony Analysis}

Harmony analysis is arguably one of the most sophisticated tasks that musicians deal with, and a formidable challenge for Sequential Learning, in that: $i$ ) it can be naturally cast to a sequential problem; ii) an intuitively neat separation between horizontal (that refers to the musical flow) and vertical (that pertains simultaneous sounds) features exists; iii) $K$ (the number of labels) is over one hundred, thereby pointing out a typical case where the Viterbi algorithm shows bad time performance.

Analyzing music harmony consists in associating a label to each vertical (that is, set of simultaneous notes) [1]. Such labels explain which harmony is sounding, by indicating a chord name through a fundamental note (root) and a mode, such as $\mathrm{C}$ minor. Given a score in MIDI format, we individuate sets of simultaneous notes (verticals or events), and associate to each vertical a label composed by 〈fundamental note, mode〉 (Fig. 1).

Music analysis task can be naturally represented as a Machine Learning classification problem, suitable to be solved by SSL techniques. In fact, by considering only the "vertical" aspects of musical structure, one would hardly produce reasonable analyses. Experimental evidences about human cognition reveal that in order to disambiguate unclear cases, composers and listeners refer to "horizontal" features of music as well: in these cases, context plays a fundamental

\footnotetext{
${ }^{1}$ In cases where the vertical rewards range over a limited interval, one could reduce the $K \log (K)$ factor to $K$ by using standard techniques [10, Chap. 9]. However, in the present application, this amounts to trade space for speed.
} 


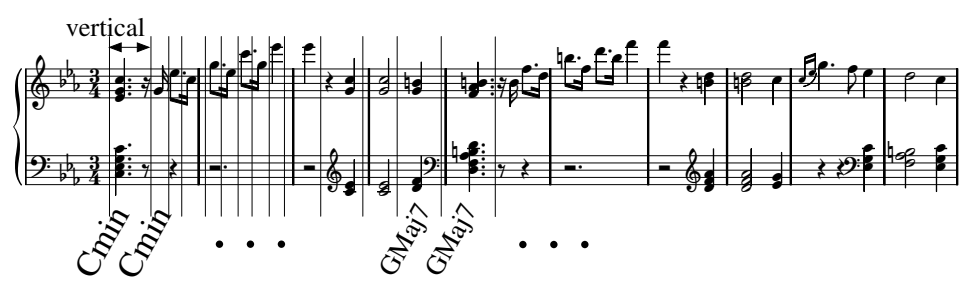

Fig. 1: The tonal harmony analysis problem consists of indicating for each vertical which chord is currently sounding.

role, and contextual cues can be useful to the analysis system. Moreover, harmony changes are well known to follow patterns where analysis must take into consideration the succession of chords (e.g., the case of cadence).

Let us go back to the SSL definition: in the case of music analysis, each $X_{m}$ corresponds to a particular piece of music; $x_{m, t}$ is the information associated to the event at time $t$; and $y_{m, t}$ corresponds to the chord label (i.e., the chord root and mode) associated to the event sounding at time $t$. The problem is, thus, to learn how to predict accurately the chord labels given the information about musical events.

By definition, a feature $\phi_{s}\left(X, y_{t}, y_{t-1}\right)$ is a boolean function of the entire sequence and of labels $y_{t}$ and $y_{t-1}$. Usually, it analyzes a small neighborhood of the current event, and returns 1 if there is evidence that the currently predicted label is correct. For the present application, the features have been engineered so that they take into account the prescriptions from Music Harmony Theory, a field where vertical and horizontal features naturally arise. Vertical features report about simultaneous sounds and their correlation with the currently predicted chord. Horizontal features capture metric patterns and chordal successions.

\subsection{CarpeDiem's performance compared with Viterbi}

We briefly recall some results of previous experimentation showing that we obtain significant running time improvements w.r.t. Viterbi. CarpeDiem has been embedded in a SSL system implementing the HMPerceptron learning algorithm [5]. The learning system has been trained iterating ten times on a data set composed of 30 chorales by J.S. Bach (1675-1750). The learnt weights have been then used to build two classifiers: one based on standard Viterbi, the other one based on CarpeDiem. The two classifiers have been fed with 42 testing sequences (chorales from the same author), their running time has been recorded and is reported in Figure 2 and in the following.

Needless to say, being equal their results, both algorithms provided the same accuracy results (on average, $79 \%$ accuracy rate). The Viterbi based HMPerceptron took 62,333 seconds of CPU time in order to complete the learning step. Performing the learning task with the CarpeDiem-based HMPerceptron took only 10,866 seconds, with a net saving of $82.56 \%$. Figure 2 reports the time spent by 


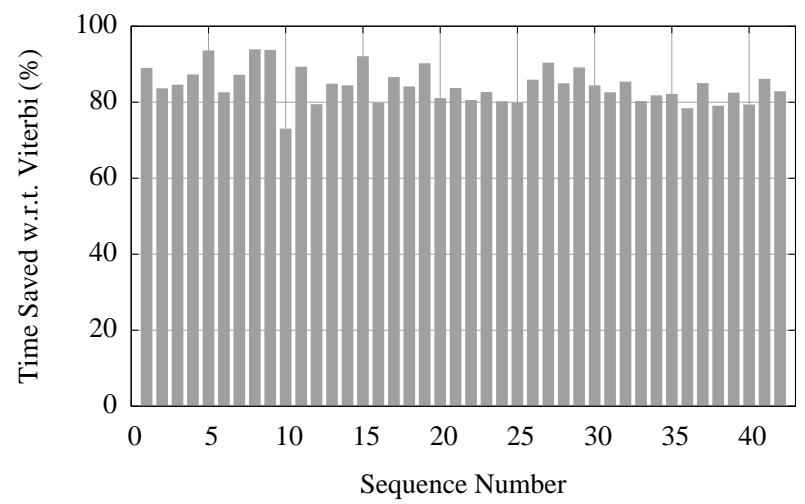

Fig. 2: Percentages of time saved by CarpeDiem w.r.t. Viterbi in analyzing the test set.

both algorithms to analyze each sequence of the test set. On average, $79 \%$ of the testing time has been saved. In the best case CarpeDiem ran in 7 seconds instead of 77 , thus saving $90 \%$ of the time. In the worst case it ran in 37 seconds instead of 118 , thus saving $68.64 \%$ of the time. Also, the magnitude of the improvement does not show large variations depending on the sequences, thus encouraging the generalization of these results to new musical pieces.

CarpeDiem clearly improves on the Viterbi algorithm on all tested sequences. However, an interesting facet of the algorithm behavior is hidden in the reported graphs: the time spent in classifying varies according to the number of sequences and the number of iterations performed.

We therefore study how the performance of the algorithm varies as important learning parameters (the training set size and the number of iterations) change. As we will see, the CarpeDiem algorithm spends more time evaluating classifiers acquired on larger datasets or by allowing more iterations of the HMPerceptron.

\section{The growth of classification time}

In order to corroborate our preliminary observations, we set up a systematic experimentation where we recorded the running time of CarpeDiem. Here, the classifiers were acquired on data sets of increasing size and using an increasing number of learning iterations. More specifically, we recorded the learning and classification times of a learning system based on CarpeDiem on nine training sets of size increasing from one to nine sequences. On each training set, we acquired ten classifiers by varying (from one to ten) the number of iterations of the HMPerceptron algorithm. We thereby obtain 90 classifiers. In each experiment, we recorded both the time needed for training the classifier and the time spent in classifying the independent test set, composed by the 42 sequences earlier mentioned (see Section 2.2).

In the following we will indicate each one of the 90 classifiers by using two digits separated by a colon: the first digit corresponds to the number of sequences 


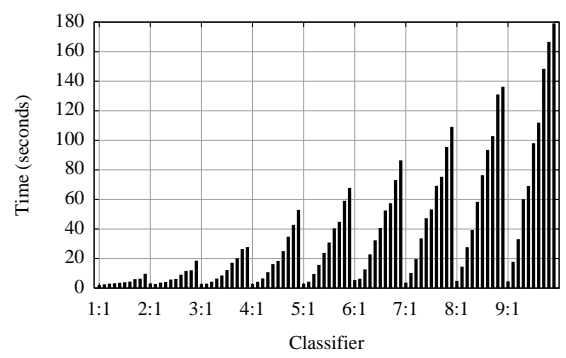

(a) Learning Times

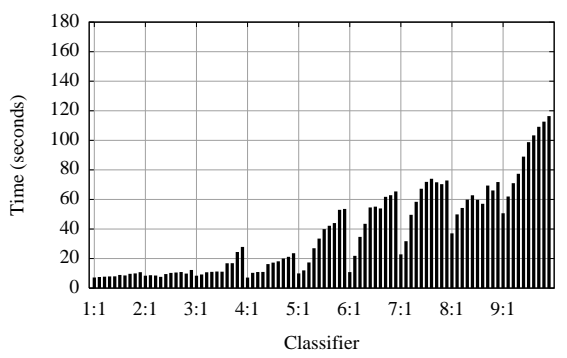

(b) Classification Times

Fig. 3: Timings versus increasing training set size and increasing number of iterations.

in the training set, the second one indicates the number of iterations. For instance, the classifier 8:7 is trained on 8 sequences with 7 learning iterations, while the classifier 5:10 corresponds to the classifier acquired on 5 sequences with 10 iterations.

Figure 3 reports training and test times ${ }^{2}$. Let us consider the behavior of learning time first. The Figure 3(a) shows a largely anticipated pattern: classifiers $m: n$ take increasing time as $m$ and $n$ grow. Much more interesting is, on the contrary, the shape of the classification time. Since classification is performed on a dataset of fixed size (the dataset is the same for all classifiers), one would expect a roughly constant classification time. On the contrary, the observed pattern looks similar to the one observed for learning times. This not only comes as a surprise, but casts in a new light the interpretation of the former graph: likely, the increase in learning times should not have been as steep as the one observed. We remark that the reported growth of testing times are to be imputed to the weights used by the classifiers. Then, as the HMPerceptron learns, it somehow fiddles with the weights in a way that proves to be detrimental to the work of CarpeDiem. Moreover, if we take a look at Figure 4, we see that all this tinkering does not provide any significant benefit as regards as the classification accuracy.

We implemented the above observation by re-running the above experiment and stopping the execution after the first iteration. Not only the results reveal a further improvement in the percentage of time saved by CarpeDiem (from $79 \%$ to $88 \%$; see Figure 5), but also the generalization accuracy increases from $79 \%$ to $81.5 \%$. Thus, it is preferable to stop the learning process much sooner than we did in 9 .

Notwithstanding the improvement in the performances of the algorithm, we are still cue-less about why the internal working of the HMPerceptron impacts so crucially on the execution of CarpeDiem.

$\overline{2}$ On a machine equipped with $2.16 \mathrm{GHz}$ Intel Core 2 Duo processor. 


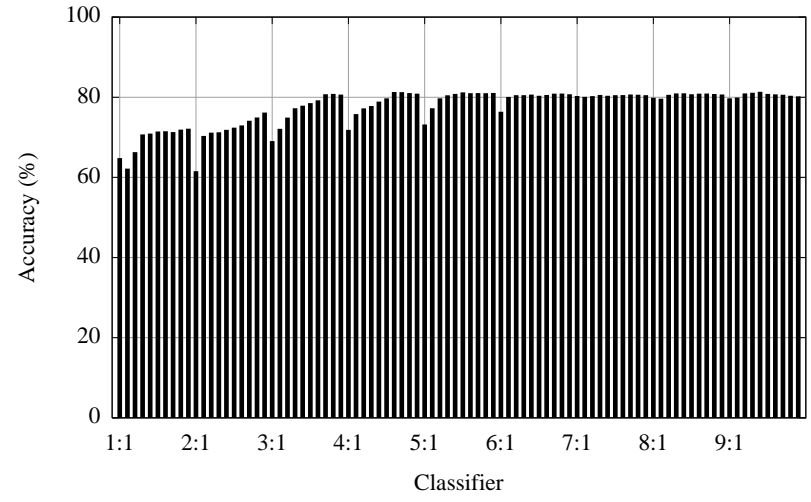

Fig. 4: Accuracies of the classification versus increasing training set size and increasing number of iterations.

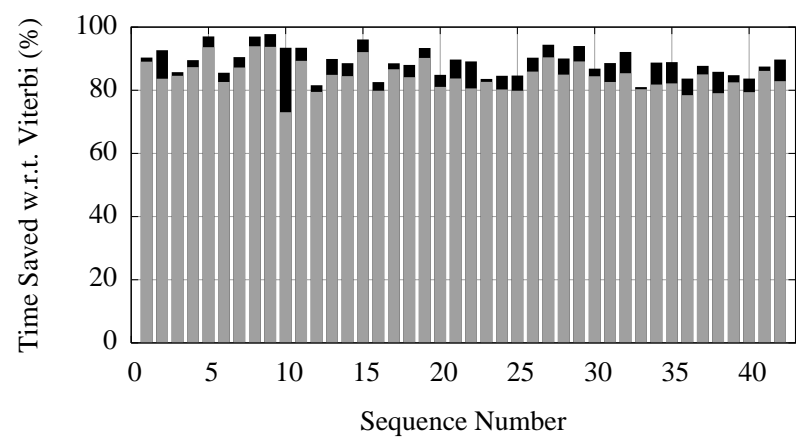

1 Iterations 10 Iterations

Fig. 5: Percentages of times saved over Viterbi by CarpeDiem (1 iteration) and CarpeDiem (10 iterations) in analyzing the test set. 


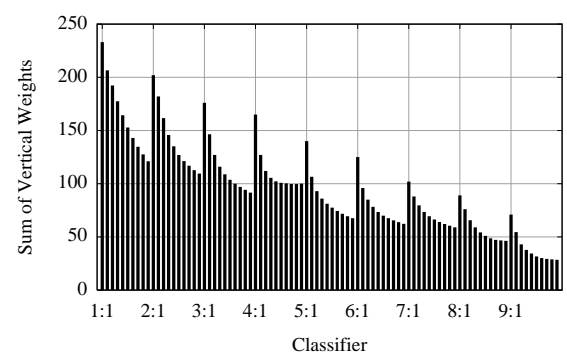

(a) Sum of vertical weights

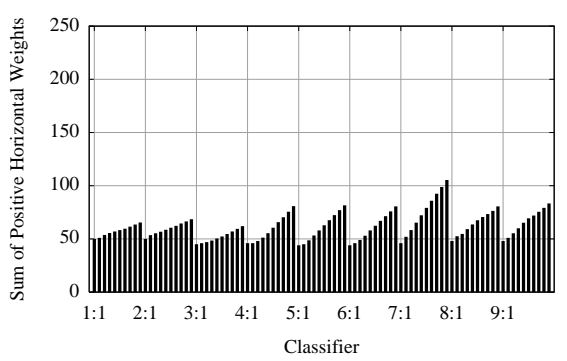

(b) Sum of positive horizontal weights

Fig. 6: Evolution of learned weights for increasing number of sequences and number of iterations.

CarpeDiem is very efficient when two conditions are met: $i$ ) vertical features are sufficient to discriminate among labels (i.e., few distinguished labels have very high rewards), and/or ii) horizontal features do not provide significant cues (i.e., most transition weights are close to the sum of positive horizontal weights). Then, two parameters that are relevant to the performances of CarpeDiem are the sum of vertical weights, and the sum of positive horizontal weights. Figure6 6 illustrates their evolution as more sequences/iterations are added.

One interesting facet of the reported graph is the distinguished pattern followed by horizontal and vertical weights. This is somehow surprising, since the weights are learnt by the HMPerceptron which is totally unaware of the distinction between horizontal and vertical weights. In a subtle though evident way, the HMPerceptron grasps the difference. In order to work out this unexpected behavior, we briefly recall few details about the learning strategy implemented by the HMPerceptron.

\subsection{HMPerceptron learning strategy}

The hypothesis acquired by the HMPerceptron has the form:

$$
H(X)=\arg \max _{Y^{\prime}=\left\{y_{1}^{\prime}, \ldots, y_{T}^{\prime}\right\}} \sum_{t} \sum_{s} w_{s} \phi_{s}\left(X, y_{t}, y_{t-1}^{\prime}\right)
$$

where $\phi_{s}$ is a boolean feature and $\left\{w_{s}\right\} \stackrel{\text { def }}{=} W$ are the weights being estimated. The HMPerceptron applies a simple scheme to optimize $w_{s}$ values: it iterates over the training set updating the weights so that features correlated to "correct" outputs receive larger values, and those correlated with "incorrect" ones receive smaller values.

This is the same kind of strategy adopted by Rosenblat's perceptron algorithm [12], the only real difference between the two algorithms is in the way the hypothesis is evaluated. In the classification problem faced by the perceptron algorithm, in fact, it is sufficient to enumerate all the possible labels and 
to pick the best one. In the case of the SSL problem, however, this cannot be done efficiently: the labelling of a single "example" is a sequence of $T$ labels, the number of such labellings is thus exponential in $T$. To overcome this problem, the algorithm uses Viterbi decoding to pick the best labelling under a first order Markov assumption.

At the beginning $W$ is initialized to the zero vector; then, for each example $\left(X_{m}, Y_{m}\right)$ in the training set, $H(X)$ is evaluated using the current $W$. Two situations may occur: the sequence of labels predicted by $H$ is identical to $Y_{m}$ or this is not the case, and a number of errors are committed. In the first case, nothing is done for that example, and the algorithm simply jumps to the following one. In the second case, the weight vector is updated by means of the following update rule

$$
w_{s}=w_{s}+\sum_{t=1}^{T} \phi_{s}\left(X, y_{t}^{\prime}, y_{t-1}^{\prime}\right)\left(I_{y_{t}^{\prime}=y_{t}}-I_{y_{t}^{\prime} \neq y_{t}}\right)
$$

where $\phi_{s}$ represents a feature, $y_{t}$ represent the $t$-th "correct label", $y_{t}^{\prime}$ denotes currently predicted $t$-th label, and $I_{P}$ represents the function that returns 1 in case $P$ is verified and 0 otherwise. By noticing that (we omit the arguments of $\phi_{s}$ for brevity):

$$
\phi_{s} \cdot\left(I_{y_{t}^{\prime}=y_{t}}-I_{y_{t}^{\prime} \neq y_{t}}\right)= \begin{cases}+1 & \text { if } \phi_{s}=1 \wedge y_{t}^{\prime}=y_{t} \\ -1 & \text { if } \phi_{s}=1 \wedge y_{t}^{\prime} \neq y_{t} \\ 0 & \text { if } \phi_{s}=0\end{cases}
$$

it is immediate to verify that the rule emphasizes features $\phi_{s}$ which are positively correlated with good classification and de-emphasize those that are negatively correlated with it.

\subsection{Trends in Vertical and Horizontal Weights}

We now come back to the problem of interpreting Figure 6. In building the CarpeDiem algorithm we have been guided by the intuition that vertical information is indeed very discriminative. Let us focus on an informative vertical feature $\phi_{\bullet}$ and consider the first iteration of the HMPerceptron on a sequence having length $T$. Just to give a bit of concreteness to our example, we assume $T=100$, that $\phi_{\bullet}$ is asserted 60 times, and that it votes for the correct label 50 times out of 60 . This example may seem very unrealistic, but it is not ${ }^{3}$. Since at the first iteration on the first sequence all labels are chosen at random, the vast majority of them will be incorrectly predicted, thus implying a large number of updates. If all the labels for which $\phi_{\bullet}$ is asserted are actually mislabelled, due to the way the update rule acts, the weight associated to $\phi_{\bullet}$ will be increased by 40 in this step. This large increase occurs all at once at the end of the first

\footnotetext{
${ }^{3}$ Some of the high level features we plugged in our Musical Analysis system work in a similar setting (for instance, this could be the case for the feature that votes for the chord that has exactly 3 notes asserted in the current event).
} 


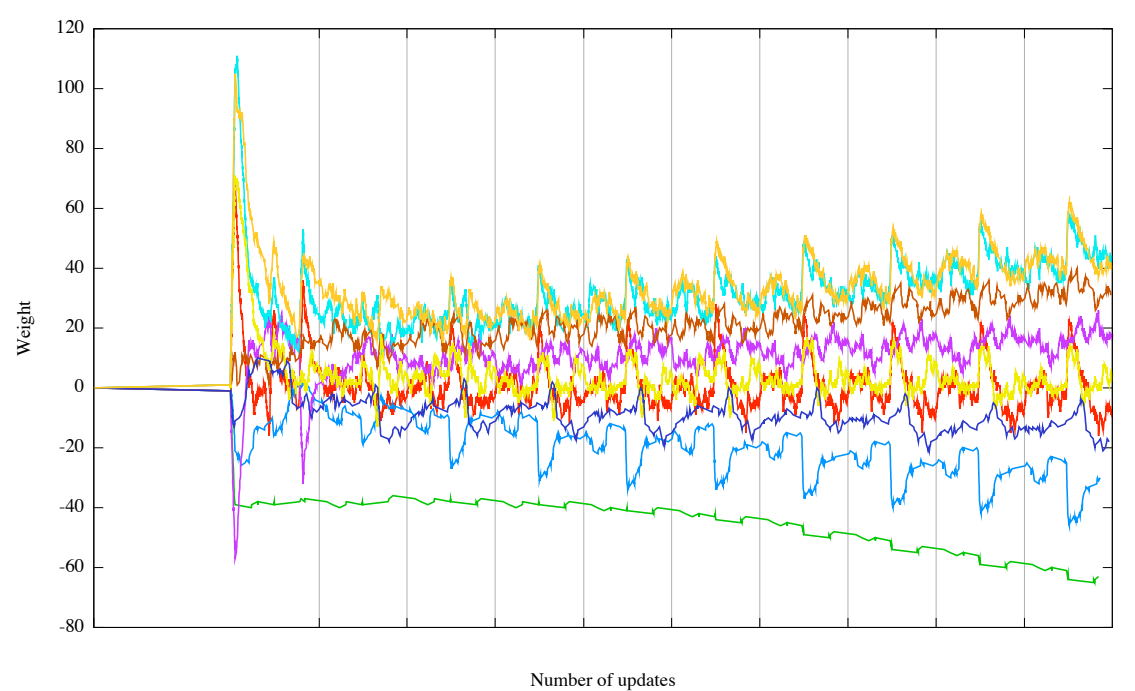

Fig. 7: Evolution of vertical weights throughout learning. Each line corresponds to an individual vertical feature; vertical lines correspond to the beginning of new iterations.

iteration on the first sequence, it is likely to overestimate the "true" weight of $\phi_{\bullet}$, and the HMPerceptron will spend the rest of learning trying to compensate for this overestimation. However, subsequent updates will be of smaller magnitude. In fact, the following predicted labeling will not be randomly guessed, thus implying a reduced number of updates. By summarizing, very predictive features have their weights initially set to very large values; such weights slowly decrease in the following. The behavior described clearly emerges in Figure 7, where the individual weights of vertical features are plotted as the updates occur. Some of the features have moderately highly negative weights. Since higher vertical weights are expected to improve the performances of CarpeDiem, one wonders if there is any means of increasing that weights without hindering the classification accuracy. To these ends, by building on the boolean nature of the features we propose a technique that we call the "inversion trick".

The assertion of a positive boolean feature can be naturally interpreted as a way to indicate which candidate labels are good. By converse, if we invert the weight of the feature and we swap its answers, the resulting feature indicates bad candidates instead of good ones. The meaning and the contribution of the feature do not change, what changes is only the way they interact with the system. Unfortunately, the inversion trick does not work with vertical features. Let us introduce the following bits of notation, and consider the inequality tested by CarpeDiem when cutting the search [9]. We denote with $\omega_{y_{t-1}^{\prime}}$ the best path found until label $y^{\prime}$ at time $t-1$; with $\Sigma_{\bar{y}_{t}, y_{t-1}^{\prime}}^{1}$ the sum of horizontal weights of 
features that vote in favour of the transition between $\bar{y}_{t}$ (our best candidate so far) and $y_{t-1}^{\prime}$ the best previous label; $\Sigma_{\bar{y}_{t}}^{0}$ the sum of vertical features that vote for $\bar{y}_{t}$; with $\omega_{t-1}^{*}$ the best of all paths to time $t-1$; and with $\Sigma^{1 *}$ the sum of positive horizontal features. The inequality tested by CarpeDiem in order to cut the search is:

$$
\omega_{y_{t-1}^{\prime}}+\Sigma_{\bar{y}_{t}, y_{t-1}^{\prime}}^{1}+\Sigma_{\bar{y}_{t}}^{0}>\omega_{t-1}^{*}+\Sigma^{1 *}+\Sigma_{y_{t}^{\prime \prime}}^{0}
$$

Since vertical weights appear on both ends of the inequality, the improvement on one side (i.e., on $\Sigma_{\bar{y}_{t}}^{0}$ ) is likely to be counterbalanced by an improvement on the other side (i.e., on $\Sigma_{y_{t}^{\prime \prime}}^{0}$ ). Interestingly enough, however, this does not hold for horizontal features. If we succeed in decreasing the weight of the vertical features it may happen that the decrease in $\Sigma^{1 *}$ is not counteracted by a decrease in $\Sigma_{\bar{y}_{t}, y_{t-1}^{\prime}}^{1}$. Guided by this intuition we tried a preliminar experiment in which we tinkered with the horizontal weights so that all positive horizontal weights were inverted, thus obtaining $\Sigma^{1 *}=0$. With our great surprise, a large decrease in CarpeDiem time performance was observed.

We are still unaware about why this happens, but the system seems to behave in a chaotic way: little changes with the inversion trick produce large variations in the running times. However, solid evidence advocates for the possibility of overcoming the mentioned issues by means of the inversion trick. First and foremost, the results we obtained so far show that the inversion trick on horizontal features indeed produces relevant changes in the running time of the algorithm without changing the learnt hypothesis. Secondly, we are currently using 30 different features in our system, resulting in about 1 billion of possible inversions configurations. The design of the features in our system has been done by focusing more on their cognitive meaning than on whether they were appropriate to the ends of improving performances by means of the inversion trick.

Basing on purely statistical accounts, one would then expect that a configuration of inversions exists that further enhances the performances of our algorithm. To efficiently find the correct inversions involves solving a new optimization problem in the space of inversions. Heuristics and algorithms for searching in this space are problems that we leave open for future work.

\section{Conclusion}

In this paper we have discussed previous relevant results, raising interesting issues about the performances of a recently proposed algorithm which improves on the Viterbi algorithm. By starting from an unexpected correlation between CarpeDiem running times and the overall amount of learning allowed, we delved into the internal working of the HMPerceptron. We showed that this phenomenon is connected with the evolution of vertical and horizontal weights as learning proceeds. In particular, vertical weights seem to be doomed to decrease while

${ }^{4}$ This can actually happen, but the effect is likely to be less systematic since on one hand we have the single horizontal weight $\Sigma_{\bar{y}_{t}, y_{t-1}^{\prime}}^{1}$, on the other hand there is the sum of all positive horizontal weights. 
horizontal weights slightly grow. We have then approached the evolution of the vertical weights. We confirmed our hypothesis about their relevance to sequential learning. To enhance the time performances of the HMPerceptron, we have considered adjusting both vertical and horizontal weights, by means of what we called inversion trick. This is influential to the run-time performances of CarpeDiem and does not affect the accuracy of the HMPerceptron. The trick is arguably relavant to improve even more CarpeDiem performances. However, it also emerged that finding the optimal configuration for (the inversions of) the features leads to a novel, difficult, optimization problem.

\section{References}

1. Dietterich, T. In: Machine Learning for Sequential Data: A Review. Volume 2396 of Lecture Notes in Computer Science. Springer-Verlag (2002) 15-30

2. Rabiner, L.R.: A tutorial on Hidden Markov Models and Selected Applications in Speech Recognition. In: Proceedings of the IEEE. Volume 77., San Francisco, CA, USA, Morgan Kaufmann Publishers Inc. (February 1989) 267-296

3. McCallum, A., Freitag, D., Pereira, F.: Maximum entropy Markov models for information extraction and segmentation. In: Proc. 17th International Conf. on Machine Learning, Morgan Kaufmann, San Francisco, CA (2000) 591-598

4. Lafferty, J., Pereira, F.: Conditional random fields: Probabilistic models for segmenting and labeling sequence data. In: Proceedings of the Eighteenth International Conference on Machine Learning, San Francisco, CA, Morgan Kaufmann (2001) 282-289

5. Collins, M.: Discriminative training methods for hidden markov models: Theory and experiments with perceptron algorithms. In: Proceedings of the Conference on Empirical Methods in Natural Language Processing. (2002)

6. Viterbi, A.J.: Error Bounds for Convolutional Codes and an Asymptotically Optimum Decoding Algorithm. In: IEEE Transactions on Information Theory. Volume 13. (1967) 260-269

7. Felzenszwalb, P.F., Huttenlocher, D.P., Kleinberg, J.M.: Fast Algorithms for LargeState-Space HMMs with Applications to Web Usage Analysis. In: Advances in Neural Information Processing System. (2003)

8. Radicioni, D.P., Esposito, R.: Tonal Harmony Analysis: a Supervised Sequential Learning Approach. In Pazienza, M.T., Basili, R., eds.: AI*IA 2007: Advances in Artificial Intelligence, 10th Congress of the Italian Association for Artificial Intelligence, Springer-Verlag (2007)

9. Esposito, R., Radicioni, D.P.: CarpeDiem: an Algorithm for the Fast Evaluation of SSL Classifiers. In: Proceedings of the 24th Annual International Conference on Machine Learning (ICML 2007). (2007)

10. Cormen, T.H., Leiserson, C., Rivest, R.L.: Introduction to Algorithms. MIT Press, Cambridge, MASS (1990)

11. Pardo, B., Birmingham, W.P.: Algorithms for chordal analysis. Computer Music Journal 26 (2002) 27-49

12. Rosenblatt, F.: The perceptron: A probabilistic model for information storage and organization in the brain. Psychological Review (Reprinted in Neurocomputing (MIT Press, 1998)) 65 (1958) 386-408 